\title{
Correction to: A bootstrapped Malmquist index applied to Swedish district courts
}

\author{
Pontus Mattsson $^{1} \cdot$ Jonas Månsson $^{1,2,3}$ (iD $\cdot$ Christian Andersson $^{2}$ • \\ Fredrik Bonander ${ }^{2}$
}

\section{Correction to: Eur J Law Econ https://doi.org/10.1007/s10657-018-9582-y}

The original version of this article unfortunately contained a mistake. Equation 7 is incomplete in the PDF version of this article. The complete Eq. 7 is given below.

The original article has been corrected.

The original article can be found online at https://doi.org/10.1007/s10657-018-9582-y.

Jonas Månsson

jonas.mansson@lnu.se

Pontus Mattsson

pontus.mattsson@1nu.se

Christian Andersson

christian.andersson@ riksrevisionen.se

Fredrik Bonander

fredrik.bonander@riksrevisionen.se

1 Department of Economics and Statistics, Linnaeus University, 35195 Växjö, Sweden

2 Swedish National Audit Office (SNAO), Nybrogatan 55, 11490 Stockholm, Sweden

3 Thammasat Centre for Efficiency and Productivity Analysis, Thammasat University, Bangkok, Thailand 


$$
\begin{aligned}
\mathrm{M}^{t, t+1}\left(\mathbf{x}_{i}^{t}, \mathbf{y}_{i}^{t}, \mathbf{x}_{i}^{t+1}, \mathbf{y}_{i}^{t+1}\right)= & \left(\frac{\mathrm{D}_{C}^{t+1}\left(\mathbf{x}_{i}^{t+1}, \mathbf{y}_{i}^{t+1}\right)}{\mathrm{D}_{C}^{t}\left(\mathbf{x}_{i}^{t}, \mathbf{y}_{i}^{t}\right)}\right) \times\left(\frac{\mathrm{D}_{V}^{t+1}\left(\mathbf{x}_{i}^{t+1}, \mathbf{y}_{i}^{t+1}\right) / \mathrm{D}_{C}^{t+1}\left(\mathbf{x}_{i}^{t+1}, \mathbf{y}_{i}^{t+1}\right)}{\mathrm{D}_{V}^{t}\left(\mathbf{x}_{i}^{t}, \mathbf{y}_{i}^{t}\right) / \mathrm{D}_{C}^{t}\left(\mathbf{x}_{i}^{t}, \mathbf{y}_{i}^{t}\right)}\right) \\
& \times\left(\frac{\mathrm{D}_{C}^{t}\left(\mathbf{x}_{i}^{t+1}, \mathbf{y}_{i}^{t+1}\right) \mathrm{D}_{C}^{t}\left(\mathbf{x}_{i}^{t}, \mathbf{y}_{i}^{t}\right)}{\mathrm{D}_{C}^{t+1}\left(\mathbf{x}_{i}^{t+1}, \mathbf{y}_{i}^{t+1}\right) \mathrm{D}_{\mathrm{C}}^{t+1}\left(\mathbf{x}_{i}^{t}, \mathbf{y}_{i}^{t}\right)}\right)^{\frac{1}{2}} \\
& \times\left(\frac{\mathrm{D}_{V}^{t}\left(\mathbf{x}_{i}^{t+1}, \mathbf{y}_{i}^{t+1}\right) / \mathrm{D}_{C}^{t}\left(\mathbf{x}_{i}^{t+1}, \mathbf{y}_{i}^{t+1}\right)}{\mathrm{D}_{V}^{t+1}\left(\mathbf{x}_{i}^{t+1}, \mathbf{y}_{i}^{t+1}\right) / \mathrm{D}_{C}^{t+1}\left(\mathbf{x}_{i}^{t+1}, \mathbf{y}_{i}^{t+1}\right)} \times \frac{\mathrm{D}_{V}^{t}\left(\mathbf{x}_{i}^{t}, \mathbf{y}_{i}^{t}\right) / \mathrm{D}_{C}^{t}\left(\mathbf{x}_{i}^{t}, \mathbf{y}_{i}^{t}\right)}{\mathrm{D}_{V}^{t+1}\left(\mathbf{x}_{i}^{t}, \mathbf{y}_{i}^{t}\right) / \mathrm{D}_{C}^{t+1}\left(\mathbf{x}_{i}^{t}, \mathbf{y}_{i}^{t}\right)}\right)^{\frac{1}{2}} \\
= & \Delta \text { PureEff } \times \Delta \text { ScaleEff } \times \Delta \text { PureTech } \times \Delta \text { ScaleTech. }
\end{aligned}
$$

